\title{
Comprehensive investigation of $T$ and $B$ cell receptor repertoires in an MC38 tumor model following murine anti-PD-1 administration
}

\author{
LU ZHANG $^{1}$, I-MING WANG ${ }^{1,2}$, NICOLAS SOLBAN ${ }^{1}$, RAZVAN CRISTESCU $^{1}$, GEFEI ZENG $^{1}$ and BRIAN LONG ${ }^{1}$ \\ ${ }^{1}$ Merck and Co., Inc., Kenilworth, NJ 07033, USA
}

Received August 11, 2019; Accepted April 20, 2020

DOI: $10.3892 / \mathrm{mmr} .2020 .11169$

\begin{abstract}
The MC38 (derived from carcinogen-induced colon adenocarcinoma) tumor model is sensitive to anti-programmed cell death-1 (anti-PD-1) treatment. However, there is no comprehensive description of the $\mathrm{T}$ and $\mathrm{B}$ cell receptor (TCR, BCR) repertoires of the MC38 tumor model following anti-PD-1 treatment, an improved understanding of which is highly important in the development of anti-PD-1 immunotherapy. The present study analyzed the TCR and BCR repertoires of three types of tissue, including tumor, spleen and tumor draining lymph node (DLN) from 20 MC38 syngeneic mice receiving murine anti-PD-1 (mDX400) treatment or mouse immunoglobulin G1 (mIgG1) control treatment. To obtain enough tissues for high-throughput sequencing, samples were collected on day 8 after the start of initial treatment. The usage frequencies of seven TCR $\beta$ chain (TRB) V genes and one TRBJ gene were significantly different between mDX400- and mIgG1-group tumors. TCR repertoire diversity was significantly lower in mDX400-group tumors compared with mIgG1-group tumors, with the top 10 most frequent TCR clonotypes notably expanded in mDX400-group tumors. In addition, the proportion of high-frequency TCR clonotypes from mDX400-group tumors that were also present both in the DLN and spleen was significantly higher than that in mIgG1-group tumors. Among the highly expanded TCR clonotypes, one TCR clonotype was consistently expanded in $>50 \%$ of the mDX400-group tumors compared with mIgG1-group tumors. Similarly, one BCR clonal family was highly expanded in $>50 \%$ of mDX400-group tumor samples. The consistently expanded TCR and BCR clones were co-expanded in $29 \%$ of mDX400-group tumors. Moreover, mutation rates of immunoglobulin heavy chain sequences in the spleen within complementarity determining
\end{abstract}

Correspondence to: Dr Brian Long, Merck and Co., Inc., Building K-15, 2000 Galloping Hill Road, Kenilworth, NJ 07033, USA E-mail: brian.long@merck.com

Present address: ${ }^{2}$ Pfizer, Inc., San Diego, CA 92121, USA

Key words: murine anti-PD-1, MC38, T cell receptor, B cell receptor, clonotype region 2 and framework region 3 were significantly higher in the mDX400 group than in the mIgG1 group. The findings of this study may contribute to an improved understanding of the molecular mechanisms of anti-PD-1 treatment.

\section{Introduction}

Immunotherapy using anti-PD-1 monoclonal antibodies (mAbs) has made significant progress in the treatment of multiple types of cancers, such as melanoma, non-small cell lung cancer, head and neck cancer, and bladder cancer, by modulating the interaction between immune and tumor cells (1-4). However, there is still a substantial proportion of patients with tumors that do not benefit from the treatment (5). Robust biomarkers are essential for patient selection. In general, two categories of biomarkers associated with responses to PD-1 inhibitors have been recognized, including those indicative of tumor neoantigen burden $(6,7)$ and those related to a T cell-inflamed tumor microenvironment $(8,9)$. $\mathrm{T}$ cell receptor (TCR) sequencing provided additional insight into the responses to anti-PD-1 immunotherapy by demonstrating that a more clonal TCR repertoire at both pre- and peri-treatment time points was associated with an improved response to anti-PD-1 treatment in patients with melanoma $(10,11)$. Despite these advances, the molecular mechanisms of anti-PD-1 therapy in treating cancers remain the subject of ongoing investigation.

Anti-PD-1 mAbs function by regulating the adaptive immune response (10), the selectivity of which derives from the enormous diversity of TCRs and B cell receptors (BCRs) (12). It is commonly known that anti-PD-1 treatment can activate tumor-specific $\mathrm{T}$ cells by blocking the immune suppressive PD-1 pathway. On the other hand, in addition to T cells, PD-1 is also expressed on human and mouse B cells, the blockade of which can induce B cell activation $(13,14)$. There is a limited but growing body of evidence that supports the notion that B cells have an important role in cancer immunotherapy. Hollern et al (15) reported that B cells and T follicular helper cells act as direct mediators of immunotherapy responses in mouse models of breast cancer. Selitsky et al (16) found that the absence of an assembled BCR in pre-treatment tumor tissues was associated with poor responses to a cytotoxic $\mathrm{T}$ lymphocyte antigen-4 (CTLA-4) inhibitor in metastatic skin cutaneous melanoma. Recently, B cells within tertiary lymphoid structures were shown to promote immunotherapy 
responses in patients with metastatic melanoma and renal cell carcinoma (17). Therefore, more in-depth investigation of the effects of anti-PD-1 mAbs on TCR and BCR repertoires is crucial for the development of anti-PD-1 mAbs in precision cancer treatment.

Mouse models are widely used to investigate mechanisms of action of immunotherapy (18). Previous studies from our group and others revealed that the MC38 tumor model is highly responsive to anti-PD-1 treatment (19-22). Recently, Efremova et al (23) demonstrated that the MC38 cell line is valid for modelling hypermutated/microsatellite-instable (MSI) colorectal cancer (CRC). Consistent with the high response rates of MSI and hypermutated CRC, the MC38 model also responds well to immune checkpoint inhibitors (ICIs). The high response rates of MSI and hypermutated CRC may be due to the high number of neoantigens. To further reveal the molecular mechanisms mediating the robust treatment responses in terms of immune responses, in-depth studies of the TCR and $\mathrm{BCR}$ repertoires of patients with hypermutated/MSI CRC or the MC38 model receiving anti-PD-1 treatment are essential. However, to the best of the authors' knowledge, until now there has not been a comprehensive description of the TCR and $\mathrm{BCR}$ repertoires of patients with hypermutated/MSI CRC or MC38-bearing mice receiving anti-PD-1 treatment. To fill this gap, the BCR and TCR repertoires of three tissues, including tumor, tumor draining lymph node (DLN) and spleen, were investigated from MC38-bearing mice treated with anti-PD-1 mAbs. The findings of this study may provide further mechanistic insights into cancer therapy using anti-PD-1 mAbs.

\section{Materials and methods}

Ethics. The care and use of mice were reviewed and approved by Merck's Institutional Animal Care and Use Committee (approval no. 200321). During the study, the care and use of animals were conducted in accordance with the guidelines of the Association for Assessment and Accreditation of Laboratory Animal Care (24). For tumor cell inoculation, animals were briefly anesthetized with $1-4 \%$ isoflurane inhalation. After tumor cell inoculation, the animals were checked daily for morbidity and mortality. At the time of routine monitoring, the animals were checked for any effects of tumor growth on normal behavior, such as mobility, food and water consumption, body weight gain/loss, eye/hair matting and any other abnormal effects. The maximal tumor volume permitted was $2,000 \mathrm{~mm}^{3}$, but other criteria were also used for the determination of humane endpoints. The other criteria included $\geq 20 \%$ body weight loss, emaciation, self-induced trauma, a tumor that interferes with basic or vital functions, and a tumor that is ulcerated. Carbon dioxide inhalation was used for euthanasia and death was confirmed by cervical dislocation.

Cell culture. The murine colon adenocarcinoma MC38 cell line (Developmental Therapeutics Program Tumor Repository, Frederick National Laboratory, Frederick, USA) was grown using early passage vials. Early passage MC38 cells were maintained as a monolayer culture in DMEM (Thermo Fisher Scientific, Inc.) supplemented with $10 \%$ heat-inactivated fetal bovine serum (Thermo Fisher Scientific, Inc.) and $2 \mathrm{mM}$
L-glutamine at $37^{\circ} \mathrm{C}$ in an atmosphere with $5 \% \mathrm{CO}_{2}$. The tumor cells were subcultured twice a week. The cells growing in an exponential growth phase were harvested and centrifuged at $335 \mathrm{x} \mathrm{g}$ in a refrigerated centrifuge at $4^{\circ} \mathrm{C}$ for $5 \mathrm{~min}$ with the medium aspirated. Cell pellets were resuspended in $10 \mathrm{X}$ volume serum-free DMEM, filtered through a $70-\mu \mathrm{m}$ nylon mesh cell strainer and counted. The cell suspension was centrifuged again as above and resuspended in serum-free and phenol red-free DMEM to obtain $1 \times 10^{7}$ cells $/ \mathrm{ml}$.

Mice. A total of 40 (20 experimental $+100 \%$ extra as spare) female C57BL/6 mice were purchased from Model Animal Research Center of Nanjing University (Nanjing, China). An acclimation period of $\sim 1$ week was allowed before tumor inoculation. Mice were kept in a special pathogen-free environment in microisolator cages at constant temperature $\left(20-26^{\circ} \mathrm{C}\right)$, constant humidity (40-70\%) and on a $12 \mathrm{~h}$ light/dark cycle. Animals had free access to food and water throughout the study. At the time of MC38 cell inoculation, the animals were 7-8 weeks of age and weighed 18-22 g. Before implantation, mice were lightly anesthetized via $1-4 \%$ isoflurane inhalation. Each mouse was inoculated subcutaneously into the right lower flank with $1 \times 10^{6}$ single cells of $\geq 95 \%$ viability suspended in $0.1 \mathrm{ml}$ of DMEM (without serum and without phenol red).

Group designation and sampling. Groups were staged and treatments were started when the mean tumor volume reached $\sim 150 \mathrm{~mm}^{3}$. Based on the tumor volume and body weight, mice were randomly assigned to murine anti-PD-1 (mDX400; Merck \& Co., Inc.) treatment group or mouse immunoglobulin G1 (mIgG1; Merck \& Co., Inc.) vehicle control group. Tumor sizes were measured on day $0,2,4$ and 7 using a digital caliper. Tumor volume (V) was estimated using the formula: $\mathrm{V}=\left(\mathrm{a} \times \mathrm{b}^{2}\right) / 2$, where ' $\mathrm{a}$ ' and ' $\mathrm{b}$ ' are long and short diameters of a tumor.

On day 0 and day $5,5 \mathrm{mg} / \mathrm{kg} \mathrm{mDX} 400$ or $\mathrm{mIgG} 1$ was administered intraperitoneally to each mouse of the corresponding group. A total of three types of tissue, including tumor, DLN and spleen, were sampled from each mouse on day 8 to obtain enough samples for sequencing, which is explained as follows.

mDX400 can induce significant tumor growth inhibition in MC38-bearing mice, which is also influenced by the initial tumor volume (tumor volume at the beginning of treatment). Our preliminary efficacy studies using MC38 tumor models and the same treatment regimen (treatment every 5 days) showed that $\mathrm{mDX} 400$ significantly $(\mathrm{P}<0.01)$ inhibited tumor growth from day 2 post-initial treatment onwards, and resulted in complete response in $50 \%$ of the mice on day 20 when treatment is initiated at an initial tumor volume of $\sim 85 \mathrm{~mm}^{3}$ (Fig. 1A). However, when treatment was started at an initial tumor volume of $\sim 210 \mathrm{~mm}^{3}$, mDX400 significantly $(\mathrm{P}<0.01)$ inhibited tumor growth from day 15 onward (Fig. 1B). In the present study, treatment was initiated when the initial tumor volume reached $\sim 150 \mathrm{~mm}^{3}$ (between the sizes of 85 and $210 \mathrm{~mm}^{3}$ from preliminary studies), so significant tumor growth inhibition should begin to occur between day 2 and day 15 post-initial treatment. In the present study, as expected based on the starting tumor size, significant differences in tumor volume 

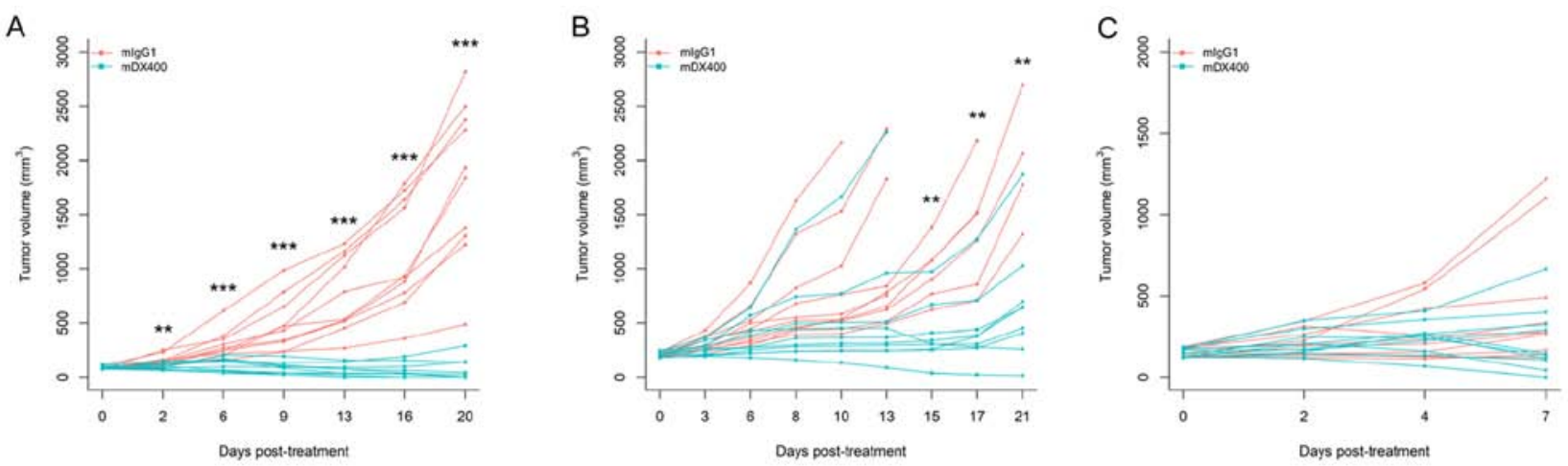

Figure 1. Tumor volume as a function of time post-initial treatment. (A) Initial tumor volume (tumor volume at the beginning of treatment) of $\sim 85 \mathrm{~mm}^{3}$. (B) Initial tumor volume of $\sim 210 \mathrm{~mm}^{3}$. (C) Initial tumor volume of $\sim 150 \mathrm{~mm}^{3}$. $\mathrm{mDX} 400$ - and $\mathrm{mIgG1}$-group tumor samples are depicted in blue and red, respectively. ${ }^{* *} \mathrm{P}<0.01,{ }^{* * *} \mathrm{P}<0.001$. $\mathrm{mDX} 400$, murine anti-PD-1; mIgG1, murine immunoglobulin G1.

were not detected between mDX400 and mIgG1-group mice on day 7 post-initial treatment (Fig. 1C). Significant differences were predicted to occur at a later time point. The mice were euthanized on day 8 to obtain enough samples for sequencing.

Library construction and sequencing. A total of $500 \mathrm{ng}$ total RNA purified using a RNeasy Mini Kit (cat. no. 74106; Qiagen, Inc.) was used for each mouse tissue sample for library construction. Quality of RNA was assessed using an Agilent 2100 Bioanalyzer (Agilent Technologies, Inc.), and samples with an RNA integrity number $<7$ were excluded from further analysis. Multiple iRepertoire primer kits with different barcodes were used to amplify the samples separately for TCR $\beta$ chain (TRB) (cat. no. MTBI-M; iRepertoire, Inc.) and immunoglobulin heavy chain (IgH) (cat. no. MBHI-M; iRepertoire, Inc.) sequences through the amplicon rescued multiplex (ARM)-PCR technology (iRepertoire, Inc.). ARM-PCR employs communal primers at the exponential phase of PCR amplification to minimize amplification biases. Library integrity was verified by gel electrophoresis and samples with abnormal profiles were excluded. After construction, the libraries were sequenced on Illumina MiSeq platform (Illumina, Inc.) using a 2x250 base pair (bp) paired-end sequencing protocol. From the 60 samples derived from the 3 tissues of the 20 mice, 54 samples were used for TCR repertoire sequencing and 52 samples were used for $\mathrm{BCR}$ repertoire sequencing, with the remaining samples excluded due to RNA degradation and abnormal library construction (Table I).

Sequence processing. Sequence quality was checked by FastQC v0.11.2 (bioinformatics.babraham.ac.uk/projects/fastqc). Paired-end reads were assembled using PANDAseq v2.10 (25) with a minimum overlap region of $30 \mathrm{bp}$. The assembled TCR and BCR reads were aligned to IMGT reference sequences (v3.1.13) (26) using IgBLAST v1.6.1 (27). Non-productive reads identified by IgBLAST were removed from further analysis.

To calculate somatic hypermutations (SHMs), BCR reads with common variable-joining (VJ) genes and identical complementarity determining region (CDR)2 to CDR3 nucleotide sequences were grouped as non-redundant reads as the forward IgH variable region primers are located within the framework region (FWR)2 region. SHMs were quantified for
CDR2 and FWR3, separately and together, with ShazaM R package v0.1.11 (28). To diminish the impact of sequencing error, only non-redundant reads with at least three copies were included in the mutation analysis.

Assignment of repertoire sequences to clones. For BCR repertoires, IgH variable-diversity-joining (VDJ) sequences were assigned into clones using 'DefineClones.py' of Change-O (28). Sequences were first partitioned into groups based on common IgH VJ genes and identical lengths of junction nucleotide sequences. Then, within each group, sequences differing from one another by a length normalized nucleotide hamming distance $<0.06$ within the junction region were clustered together as a clone. The threshold 0.06 was determined by plotting the normalized hamming distance of each sequence to its nearest neighbor in the same group (Fig. S1). If a clone contained at least two different sequences, each possessing no less than three copies, the clone was recognized as a clonal family. After obtaining clonal families in each sample independently, the sequences of clonal families from all samples were clustered together for comparison purposes. To quantify selection pressure within BCR repertoires, BASELINe implemented in ShazaM was employed to calculate the posterior distribution based on observed mutation rates and expected mutation rates derived from a default human 5-mer mutation model. Selection strengths in CDR2 and FWR3 were calculated by using an effective sequence for each clone.

For TCR repertoires, a clone was represented as the combination of a TRB V, J gene and a CDR3 amino acid sequence. VJ usage and CDR3 lengths of BCR/TCR repertoires were calculated using BCR/TCR clones with clone abundance of at least three to minimize the impact of sequencing error on the calculation.

Clonal diversity analysis. For each sample, the clonal diversity of the BCR/TCR repertoire was characterized by Hill's diversity indices (29) for diversity orders (q) from 0 to 30 in 0.1 increments using the rarefyDiversity function of Alakazam package (28). To eliminate the effect of variations in sequencing depth, each repertoire was randomly sub-sampled to the number of sequences in the smallest sample and the diversity indices for each sample were averaged over 1,000 sub-samples. Cross-classification based on the two treatments and three tissues resulted in six groups. For each group, the Hill diversity 
Table I. Number of tissue samples used for BCR/TCR repertoire sequencinga .

A, mDX400 group

\begin{tabular}{lccc}
\hline Immune repertoire & Tumor & Tumor draining lymph node & Spleen \\
\hline BCR & 7 & 10 & 9 \\
TCR & 7 & 10 & 9 \\
\hline
\end{tabular}

B, mIgG1 group

\begin{tabular}{lccc}
\hline Immune repertoire & Tumor & Tumor draining lymph node & Spleen \\
\hline BCR & 8 & 9 & 9 \\
TCR & 9 & 10 & 9
\end{tabular}

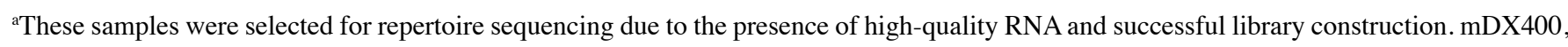
murine anti-PD-1; mIgG1, murine immunoglobulin G1; BCR, B cell receptor; TCR, T cell receptor.

index value at each $\mathrm{q}$ was obtained by calculating the mean and standard deviation.

Identification of TCR clones consistently enriched in mDX400-group tumors. To detect TCR clones consistently expanded in mDX400-group tumors compared with mIgG1-group tumors, high-frequency TCR clones with frequencies of at least $0.1 \%$ in $\mathrm{mDX} 400$-group tumors were investigated. The cutoff $0.1 \%$ was chosen because for each mDX400-group tumor, the frequencies of the top 10 most frequent TCR clonotypes were $>0.1 \%$ (Fig. S2). A TCR clone with a frequency of at least $0.1 \%$ in an mDX400-group tumor was recognized as expanded in the tumor if its frequency is at least 10 -fold larger than the mean frequency of the clone in mIgG1-group tumors. If a TCR clone was detected to be expanded in $>50 \%$ of the seven mDX400-group tumors, the TCR clone was recognized as a consistently expanded TCR clone. In addition, the presence of the consistently expanded clones in tissues other than tumor was also investigated.

Statistical analysis. Wilcoxon rank-sum test was used to compare differences between two groups and produce the P-values. Multiple comparisons were corrected by Benjamini-Hochberg procedure to control the false discovery rate (FDR). $\mathrm{P}<0.05$ and $\mathrm{FDR}<0.05$ were considered to indicate statistically significant differences in single comparisons and multiple comparisons, respectively. For each tissue, the comparisons between treatment and control groups were performed using 7-10 biological replicates per group (Table I).

\section{Results}

$V$, J usage and CDR3 lengths. For TCR repertoires, comparing tumor samples between the two treatment groups showed that frequencies of seven TRBV genes and one TRBJ gene were significantly different (FDR<0.05), including TRBV12-2, TRBV13-1, TRBV3, TRBV4, TRBV15, TRBV17, TRBV20 and TRBJ2-3, with the first two TRBV genes (TRBV12-2 and TRBV13-1) more frequent in mDX400-group tumors
(Fig. 2A and B). In addition, TRBV19 was expressed at a significantly $(\mathrm{FDR}<0.01)$ higher frequency in mDX400-group spleen samples compared with mIgG1-group spleen samples (data not shown). As for BCR repertoires, no significant differences in the usage frequencies of $\mathrm{IgHV}$ or IgHJ genes were detected between the two treatment groups derived from any of the three tissues (data not shown).

Regarding the CDR3 lengths of TCR/BCR repertoires, no significant differences were detected between the two treatment groups derived from any of the three tissues. For TCR repertoires in any tissue and BCR repertoires in DLN, the average CDR3 length is 12 amino acids. For BCR repertoires in the tumor and spleen, the average CDR3 length is 11 amino acids (Fig. S3).

Clonal diversity and tissue distribution of TCR repertoires. The intratumoral TCR repertoires of $\mathrm{mDX} 400$-treated mice had significantly lower ${ }^{\mathrm{q}} \mathrm{D}$ values $(\mathrm{FDR}<0.01)$ compared with those of mIgG1-treated mice at each q from 0 to 30, indicating lower TCR repertoire diversity of mDX400-group tumors (Fig. 3A). As diversity leveled off after $q=2$, only q-values 1-10 are presented in Fig. 3A. In contrast, there were no significant differences in the ${ }^{9} \mathrm{D}$ values of TCR repertoires in DLN or spleen tissues between the two treatment groups at any q value (Fig. S4).

To further investigate the abundance profile of intratumoral TCR clones between the two treatment groups, segmental frequency distribution of TCR clones in each tumor sample was calculated. It was found that the proportion of the top 10 most frequent TCR clonotypes in mDX400-group tumors were significantly greater $(\mathrm{P}<0.01)$ than that in $\mathrm{mIgG1}$-group tumors, with an average proportion of $46.7 \%$ vs. $5.5 \%$ (Fig. 3B). In addition, there was also a significant increase in the cumulative frequencies of the top 11-100 $(\mathrm{P}<0.05)$ and top 101-1,000 $(\mathrm{P}<0.01)$ TCR clonotypes and a significant decrease in the cumulative frequencies of $>1,000$ TCR clonotypes $(\mathrm{P}<0.01)$ by frequency in the $\mathrm{mDX} 400$-group tumor samples compared with the mIgG1-group tumor samples.

Distribution and frequencies of intratumoral TCR clonotypes in other tissues were also investigated. On average, $60 \%$ of the top 10 most frequent TCR clonotypes in mDX400-group 
A

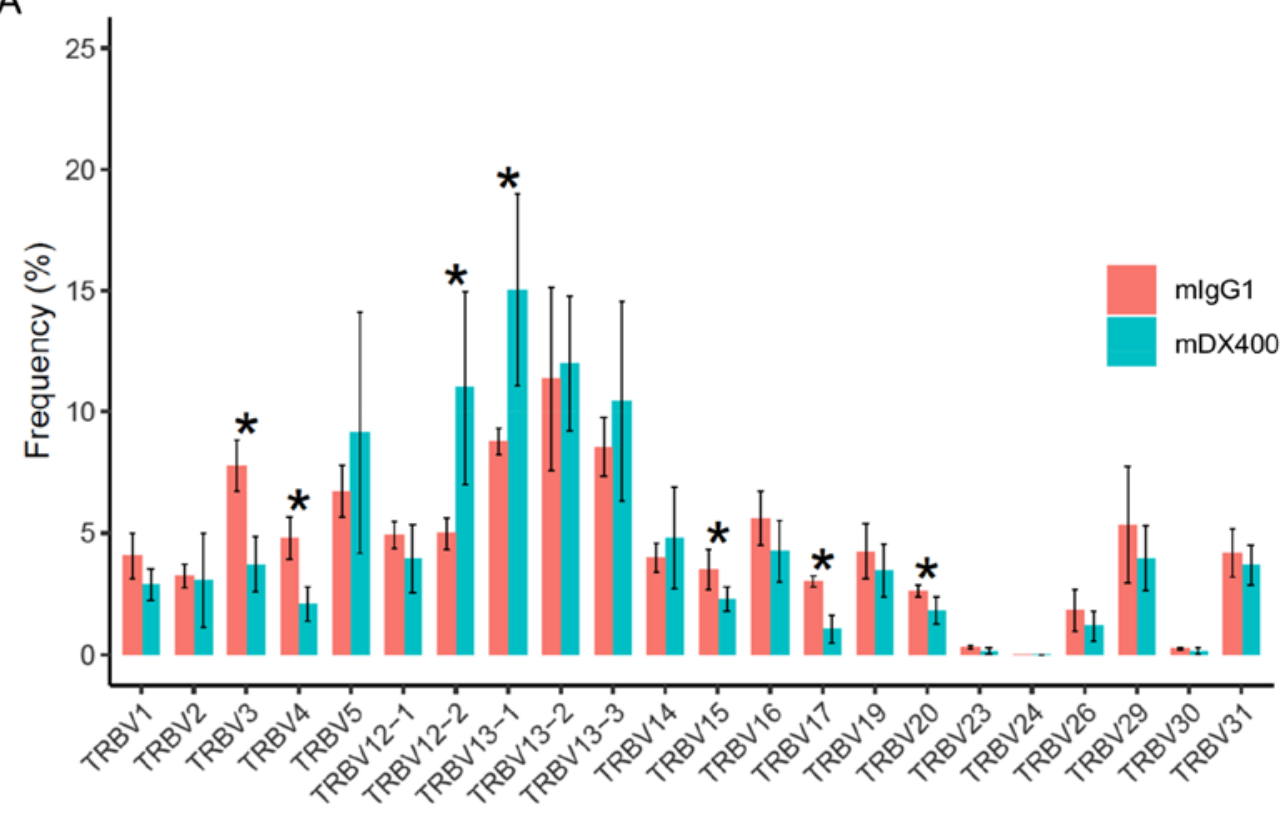

B

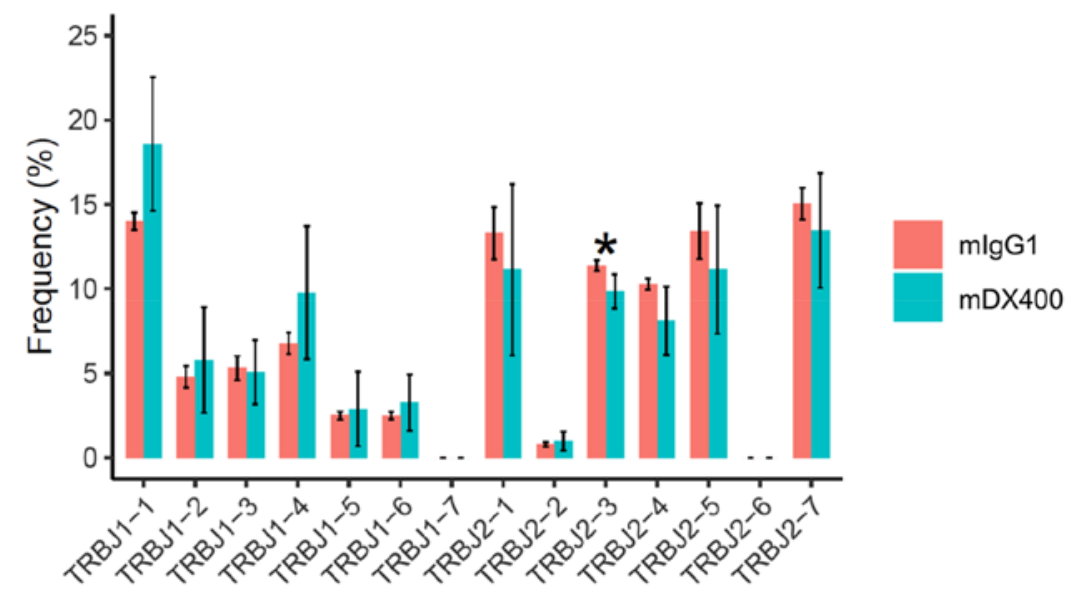

Figure 2. Usage frequencies of TCR $\beta$ chain V and J genes. (A) TCR $\beta$ chain V genes. (B) TCR $\beta$ chain J genes. mDX400- and mIgG1-group tumor samples are depicted in blue and red, respectively. Data are represented as the mean \pm standard deviation. ${ }^{*}$ FDR $<0.05$. TCR, T cell receptor; TRB, TCR $\beta$ chain; V, variable; J, joining; mDX400, murine anti-PD-1; mIgG1, murine immunoglobulin G1.

tumors can also be detected both in the spleen and DLN of the corresponding mice, which is significantly $(\mathrm{P}<0.05)$ higher than that (10\%) in mIgG1-group tumors (Fig. 3C). As to the top 10 most frequent intratumoral TCR clonotypes irrespective of treatments, the frequency of every shared TCR clonotype is higher in the tumor compared to the corresponding spleen or DLN with enrichment up to 232,829 and 35,877 folds, respectively, and mean enrichment of 4,972 and 2,073 folds, respectively. Likewise, $53 \%$ of the top 11-100 most frequent TCR clonotypes in mDX400-group tumors can also be detected in both the spleen and DLN of the corresponding mice, which is significantly $(\mathrm{P}<0.05)$ higher than that $(16 \%)$ in $\mathrm{mIgG1-group}$ tumors (Fig. 3C).

TCR clones consistently expanded in mDX400-group tumor samples. As mDX400-group tumors had significantly more focused TCR repertoires, highly frequent TCR clones consistently expanded in mDX400-group tumors were investigated.
One TCR clone with CDR3 sequence ASSPDRGDTEVF, as well as VJ genes TRBV15 and TRBJ1-1, was significantly upregulated in mDX400-group tumors compared with mIgG1-group tumors $(\mathrm{P}<0.01)$ and was expanded in four of the seven mDX400-group tumor samples (G2M119, G2M116, G2M124 and G2M120; Fig. 4). The clone was also present in the spleen and DLN of the two groups of mice, with mean frequency of $0.02 \%$ for spleen samples of both groups, as well as $0.07 \%$ and $0.009 \%$ for mIgG1- and mDX400-group DLN samples, respectively. In mDX400-group mice the frequency of the clone was significantly upregulated in the tumor samples compared with the spleen $(\mathrm{P}<0.01)$ or DLN $(\mathrm{P}<0.001)$ samples. There were no significant differences in the frequencies of the clone between the tumor and spleen samples, or between the tumor and DLN samples in mIgG1-group mice (data not shown). The clone was not recorded in databases VDJdb (June 2018 release) or McPAS-TCR (April 2019 release), which are two curated databases of known antigen-specific TCR sequences $(30,31)$. 
A

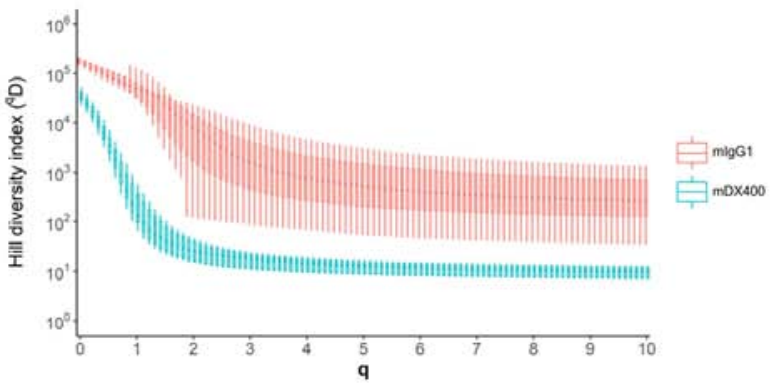

B
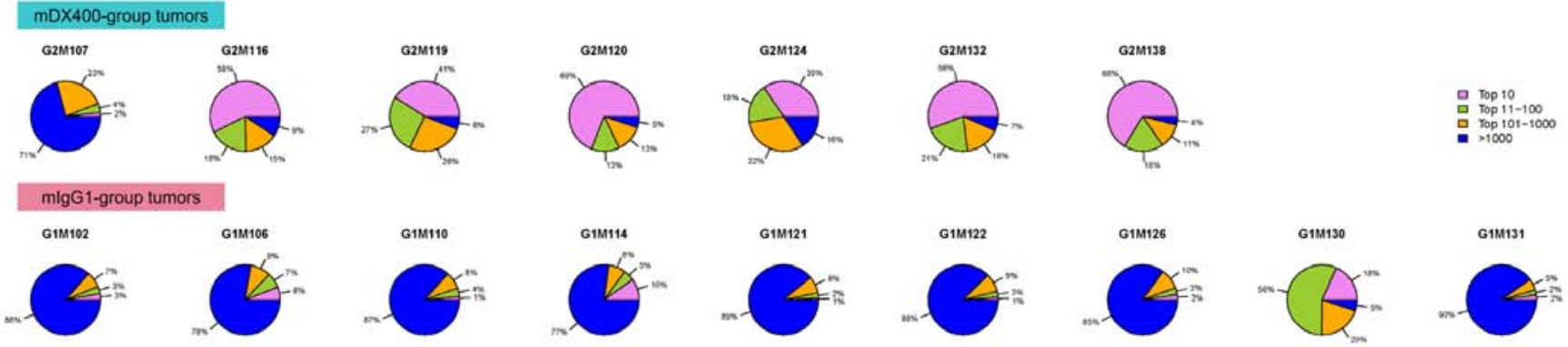

C
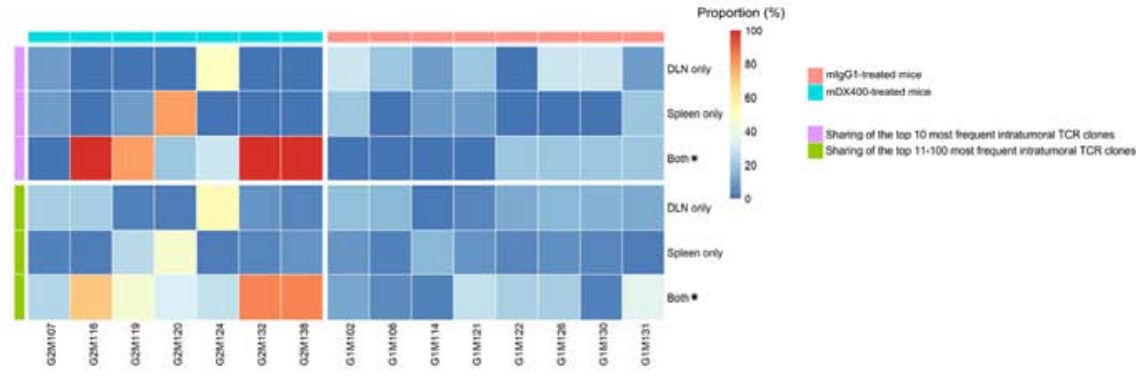

Figure 3. Clonal diversity and tissue distribution of TCR repertoires. (A) Hill diversity profiles of intratumoral TCR repertoires. mDX400- and mIgG1-group tumor samples are depicted in blue and red, respectively. The diversity profile of each group is represented by a set of boxplots with each boxplot displaying the diversity values in ${ }^{9} \mathrm{D}$ relative to a certain $\mathrm{q}$. The $\mathrm{q}$ values ranging from 0 to 10 in 0.1 increments are shown on the plot. For each $\mathrm{q}$, the Hill index value is significantly $($ FDR $<0.01)$ lower in mDX400- compared with mIgG1-group tumor samples. The FDR values are not shown on the plot. (B) Sectional proportions of intratumoral TCR clonotypes. mDX400- and mIgG1-group tumor samples are shown in the upper and lower row, respectively. For each pie chart, sections depicting the proportions of the top 10, top 11-100, top 101-1,000 and >1,000 most frequent clonotypes are depicted in violet, green, orange and blue, respectively. (C) Sharing of the most frequent intratumoral TCR clonotypes across other tissues. The plot depicts the sharing of the top 10 (marked with violet bars on the left side) and the top 11-100 (marked with green bars on the left side) most frequent intratumoral TCR clonotypes with DLN only, spleen only and both DLN and spleen. mDX400-treated mice were marked with blue bars on top, while mIgG1-treated mice were marked with red bars on top. The scale of the proportions of shared TCR clonotypes are shown in the color key. ${ }^{*} \mathrm{P}<0.05$, mIgG1 vs. mDX400. TCR, T cell receptor; mDX400, murine anti-PD-1; mIgG1, murine immunoglobulin G1; FDR, false discovery rate; DLN, tumor draining lymph node.

Mutation rate, clonal diversity and tissue distribution of $B C R$ Repertoires. To confirm that the B cells from different tissues of the MC38 tumor model were antigen-driven, selection pressure was quantified by analyzing mutation patterns in $\operatorname{IgH}$ sequences. This analysis showed evidence of negative selection in the FWR3 and positive selection in the CDR2 of all BCR repertoires, with estimated selection strength significantly different from zero in all cases $\left(\mathrm{P}<10^{-7}\right)$, except selection strength in the CDR2 of intratumoral $\mathrm{BCR}$ repertoires $(\mathrm{P}=0.16)$, which were consistent with the characteristics of affinity-matured B cells (Fig. S5). Further confirming the antigen experience of the different $\mathrm{BCR}$ repertories of the MC38 tumor model, most of the BCR sequences from different repertoires were distributed into clonal families. On average, $>98 \%$ of tumor BCR sequences were distributed into clonal families (Table SI). For spleen and DLN samples, the mean proportions of BCR sequences belonging to clonal families were $84 \%$ and $83 \%$, respectively.
Analysis of mutations showed that the number of replacement mutations per $10^{4} \mathrm{bp}$ in the CDR2, as well as the number of silent and replacement mutations in the FWR3 (or in the two regions together), were significantly higher in $\operatorname{IgH}$ sequences from mDX400-group spleen samples compared with those from mIgG1-group spleen samples $(\mathrm{P}<0.01$; Fig. 5). In addition, as presented in Fig. S6, the replacement mutation rate shows a trend of upregulation in the CDR2 of $\operatorname{IgH}$ sequences from mDX400-group tumor samples compared with those from mIgG1-group tumor samples, although this was not statistically significant.

In terms of BCR repertoire diversity, no significant differences were detected between mDX400 and mIgG1 groups derived from any of the three tissues, with no significant differences in Hill diversity indices ${ }^{9} \mathrm{D}$ values detected at any $\mathrm{q}$ from 0 to 30 between the BCR repertoires of the two groups derived from any of three tissues (Fig. S7). As 


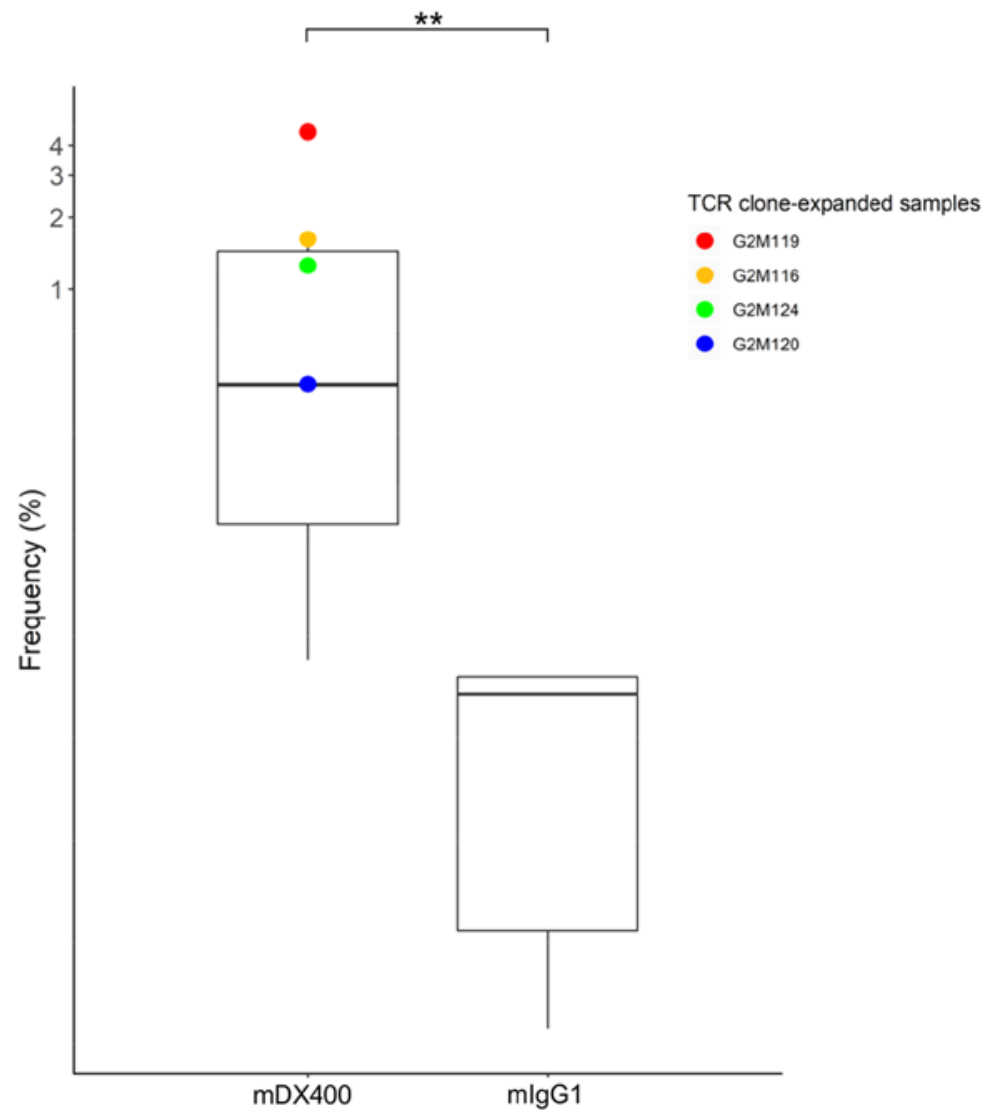

Figure 4. TCR clone consistently expands in mDX400-group tumor samples. The frequencies of the TCR clone were significantly upregulated in mDX400-group tumor samples compared with mIgG1-group tumor samples. The TCR clone was highly expanded in four mDX400-group tumor samples, which are depicted by four dots in red, yellow, green and blue, with each color representing an mDX400-group tumor sample. ${ }^{* *} \mathrm{P}<0.01$. TCR, T cell receptor; mDX400, murine anti-PD-1; mIgG1, murine immunoglobulin G1.

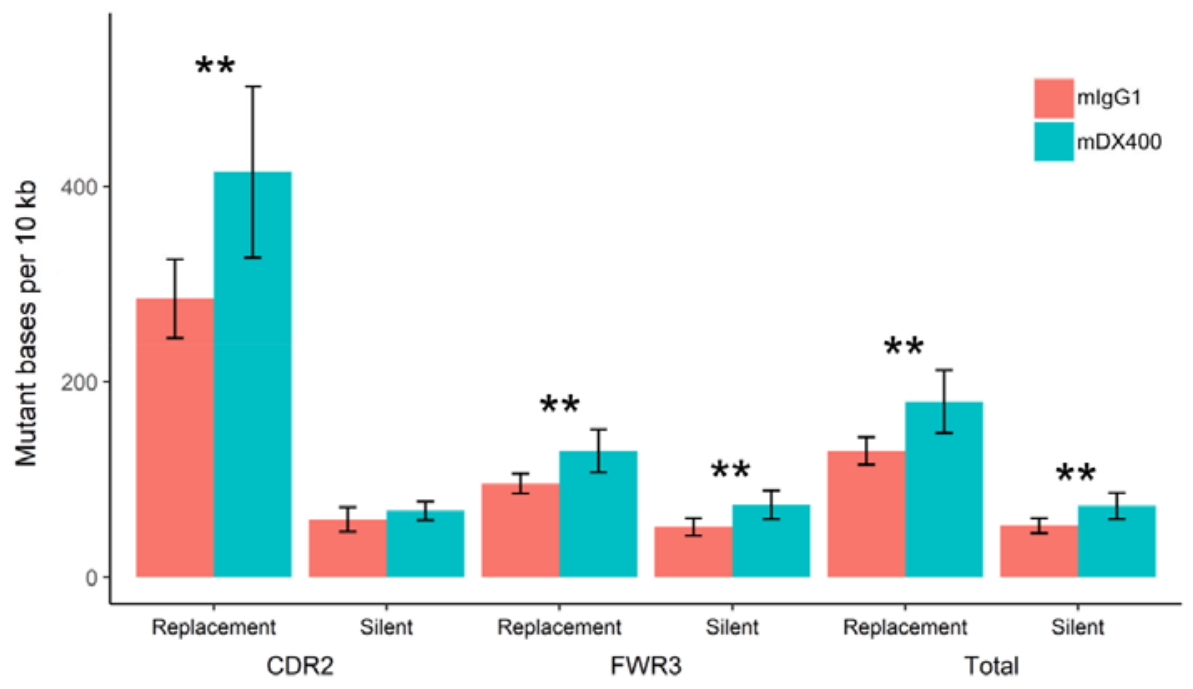

Figure 5. Mutation rates of IgH repertoires in spleen samples. Mutation rates are represented by the number of mutant bases per $10^{4}$ base pairs within CDR2 and FWR3, separately and together. mDX400- and mIgG1-group spleen samples are depicted in blue and red, respectively. Data are represented as the mean \pm standard deviation. ${ }^{* *} \mathrm{P}<0.01$. IgH, immunoglobulin heavy chain; CDR2, complementarity determining region 2; FWR3, framework region 3; mDX400, murine anti-PD-1; mIgG1, murine immunoglobulin G1.

diversity leveled off after $\mathrm{q}=2$, only q-values 1-10 are presented in Fig. S7. On the other hand, irrespective of treatments, the diversity of BCR repertoire in tumor samples was significantly lower compared with that in DLN $($ FDR $<0.01)$ and spleen samples $(\mathrm{FDR}<0.01)$ based on ${ }^{\mathrm{q}} \mathrm{D}$ values at any $\mathrm{q}$ from 0 to 30 (Fig. S8). As diversity leveled off after $\mathrm{q}=2$, only q-values 1-10 are presented in Fig. S8. Analysis of tissue distribution of BCR repertoires showed that there were no 

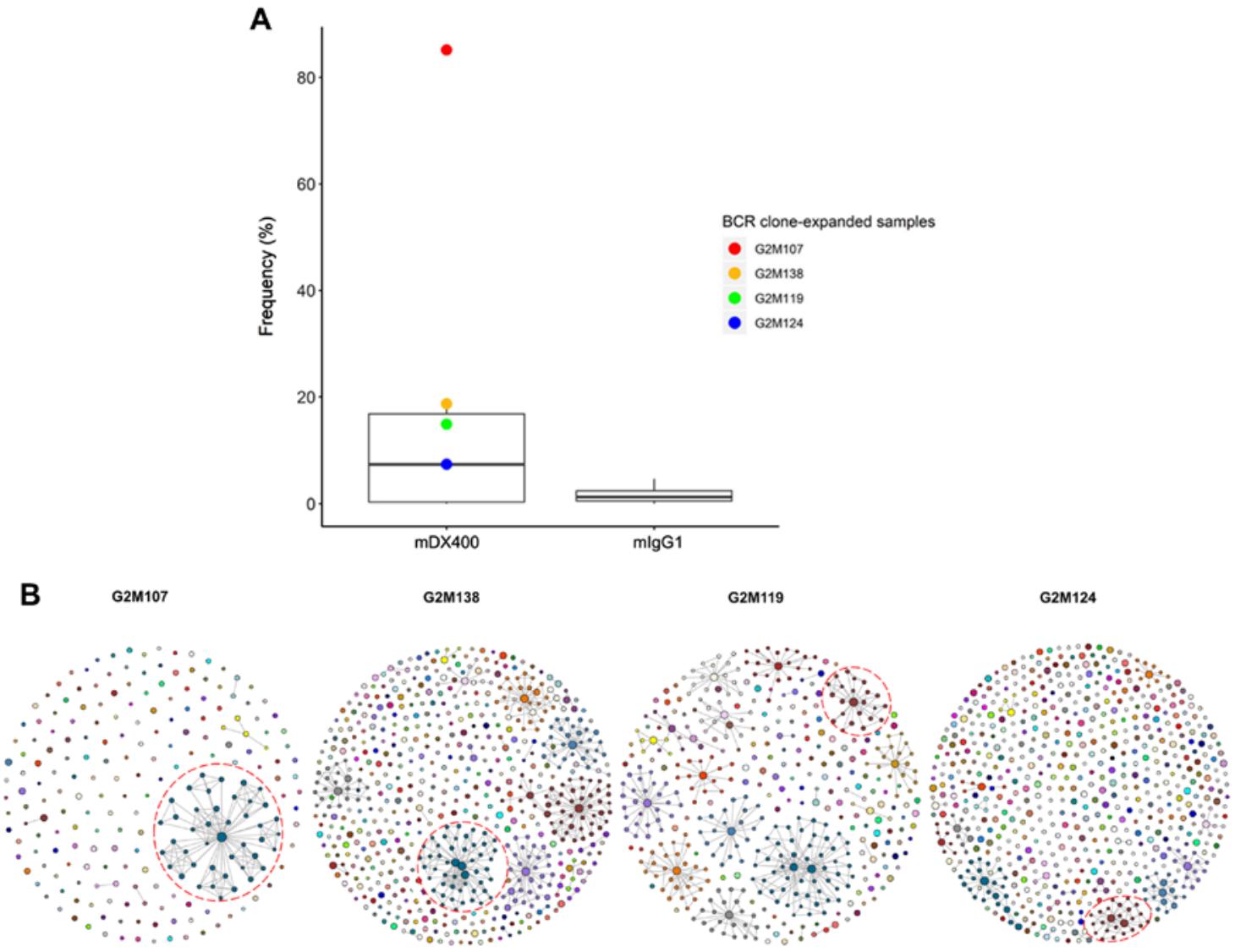

C

G2M107
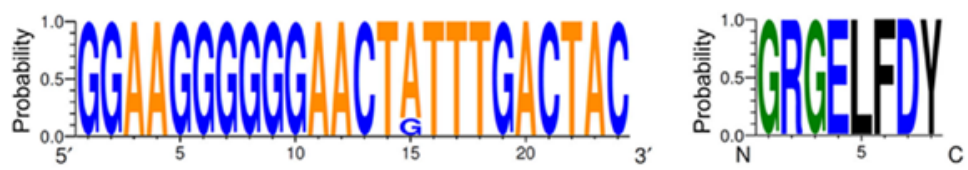

G2M119
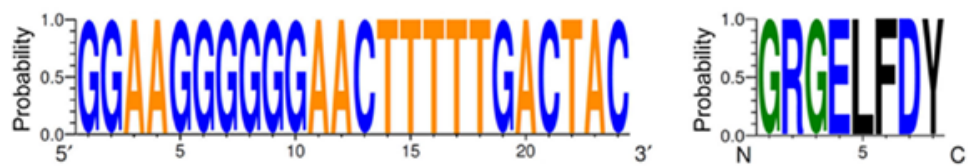

G2M124
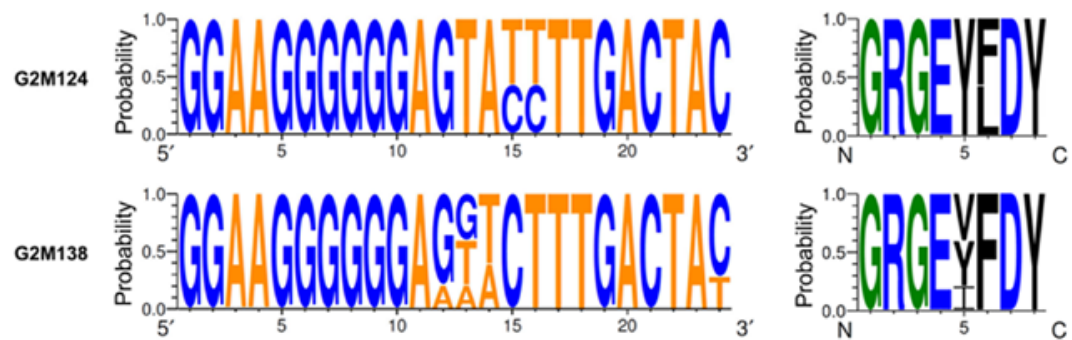

Figure 6. BCR clone consistently expands in mDX400-group tumor samples. (A) BCR clone was highly expanded in four mDX400-group tumor samples, which are depicted by four dots in red, yellow, green and blue, with each color representing an mDX400-group tumor sample. (B) Intratumoral BCR clones of the four mDX400-group tumor samples where the consistently expanded BCR clone was expanded. For each sample, a clone with a unique junctional sequence was displayed by a colored dot. A clone with $>1$ unique junctional sequence is displayed by interconnected dots of the same color. Each color corresponds to the frequency rank of a clone and the size of a dot is proportional to the abundance of the read. For each sample, the consistently expanded BCR clone is enclosed by a red dashed circle. (C) Complementarity determining region 3 nucleotide (left) and amino acid (right) composition of the consistently expanded BCR clonal family. Logos were created for each of the four mDX400-group tumor samples G2M107, G2M119, G2M124 and G2M138 where the BCR clonal family expanded. For each sequence logo, the height of symbols within a stack indicates the probability of each symbol to occur at that position. For nucleic acids, letter colors were used according to number of Watson-Crick hydrogen bonds, while for amino acids, letter colors correspond to hydrophobicity with hydrophilic, neutral and hydrophobic amino acids represented by blue, green and black colors, respectively. BCR, B cell receptor; mDX400, murine anti-PD-1; mIgG1, murine immunoglobulin G1.

significant differences in the number of intratumoral BCR clonal families shared with BCR clones in spleen or DLN tissues between the two treatment groups (Fig. S9).
Expansion of a BCR clonal family in mDX400-group tumor samples. By analyzing BCR repertoire sequences, one BCR clonal family was highly expanded in four of the seven 
mDX400-group tumor samples (Table SII). The clonal family was the most abundant clone in two mDX400-group tumor samples (G2M107 and G2M138), accounting for $85.2 \%$ and $18.7 \%$ of the total BCR sequences, respectively, and was the second most abundant clone in another two mDX400-group tumor samples (G2M119 and G2M124), constituting 14.9\% and $7.4 \%$ of the BCR repertoires, respectively (Fig. 6A and B). The mean frequency of the clonal family in mDX400-group tumors was 10 -fold greater than that of mIgG1-group tumors, and 55-470-fold higher than that of other tissue samples, although the differences in frequencies between mDX400and mIgG1-group tumors were not significant. The BCR clone and the aforementioned consistently expanded TCR clone were co-expanded in two (G2M119 and G2M124) of the seven mDX400-group tumor samples. Furthermore, in the tumor samples of six mDX400-group mice, high expansion of the top 100 most frequent TCR clonotypes were detected, except that of G2M107 (Fig. 3B). On the contrary, it was only in G2M107 that the extreme expansion, with a frequency of $85.2 \%$, of the aforementioned BCR clone could be detected (Fig. 6B), suggesting that the extreme expansion of the BCR clone may affect the expansion of the most frequent TCR clones.

The BCR clonal family contained significantly $(\mathrm{P}<0.05)$ more silent mutations within the CDR2 of $\operatorname{IgH}$ sequences from the four mDX400-group tumor samples where the clonal family expanded compared with those from the other three mDX400-group tumor samples where the clonal family was not expanded (Fig. S10). A lineage tree from the clonal family was constructed for each of the four clone-expanded mDX400-group tumor samples using the top 50 most frequent sequences of variable region stretching from the beginning of CDR2 to the start of CDR3 (Fig. S11). The germline V and $\mathbf{J}$ genes of the clonal family are IGHV1-11 and IGHJ2. The sequence logos of the CDR3 nucleotide and amino acid composition of the clonal family for each of the four tumor samples are shown in Fig. 6C.

\section{Discussion}

Previous studies demonstrated that the MC38 tumor model is highly responsive to anti-PD-1 treatment (19-22). To further understand the molecular mechanism underlying the high response rate, the present study investigated the effects of anti-PD-1 antibody on the adaptive immune receptor repertoires of the MC38 tumor model in detail, an improved understanding of which may facilitate the development of anti-PD-1 mAbs in precision medicine for cancer.

In terms of $\mathrm{T}$ cell-mediated immunity, a significant decrease in TCR repertoire diversity and a notable expansion of the top 10 most frequent TCR clones were observed in mDX400-group tumors. It has been previously reported that decreased TCR repertoire diversity at both pre- and peri-treatment time points was associated with an increased response to anti-PD-1 treatment in patients with melanoma $(10,11)$. In addition, a consistently expanded TCR clonotype was detected in mDX400-group tumors, which may be a tumor-reactive TCR clone activated and expanded by mDX400 treatment. Several studies have demonstrated that the most remarkably expanded TRB clonotypes in $\mathrm{CD}^{+}$and $\mathrm{CD} 8^{+} \mathrm{PD}-1^{+}$tumor-infiltrating lymphocyte populations are tumor- and mutated antigen-reactive in metastatic melanoma $(32,33)$. Further research is warranted to explore the interaction among the consistently expanded TCR clone, anti-PD-1 mAbs and tumor responses. It was observed that compared with mIgG1-treated mice, there were significantly more intratumoral high-frequency TCR clonotypes present both in DLN and spleen tissues of mDX400-treated mice with the shared TCR clonotypes significantly enriched in tumors. Similarly, Hosoi et al (34) also found that immunotherapy increased the number of TCR clonotypes shared between the tumor and spleen.

In addition to $\mathrm{T}$ cell responses, which are often the main focus of immunotherapy, the present study also investigated B cell responses of the MC38 model during checkpoint inhibition. Analysis of selection pressure and proportion of clonal families confirmed the antigen experience of different BCR repertoires of the MC38 model. In addition, significantly higher mutation rates were detected in the CDR2 and FWR 3 of IgH sequences from the mDX400-group spleen samples compared with those from the mIgG1-group spleen samples. The spleen is a highly active site of B cell antigen training. The significant upregulation of mutations in $\operatorname{IgH}$ sequences from spleen samples induced by mDX400 treatment suggested that mDX400 can modulate B cell immunity in the spleen. The upregulation of mutations may increase the BCR affinity, thereby enhancing antigen capture and presentation in the spleen, which may be associated with tumor responses. Notably, Lehmann-Horn et al (35) observed a more pronounced antigen-driven process in the spleen and ectopic lymphoid tissue, but not in the lymph node and blood, by examining mutational patterns of mouse BCR repertoires. This is consistent with the present finding that the mutational pattern of the spleen is different from that of the DLN.

Quantification of the BCR repertoire diversity revealed that irrespective of treatments the diversity of BCR repertoires in tumor samples was significantly lower compared with that in DLN and spleen samples, which is consistent with a previous report (36). In addition, one BCR clonal family was highly expanded in $>50 \%$ of the $\mathrm{mDX} 400$-group tumor samples, which may target public tumor antigens. In line with the present results, in the blood plasmablasts of patients with non-progressing cancer treated by anti-PD-1 or anti-CTLA-4, DeFalco et al (37) also detected B cell responses represented by increased somatic hypermutation and clonal expansion. Furthermore, they demonstrated that the anti-tumor B cell responses express shared antibody paratopes targeting public tumor antigens. In the present study, the somatic hypermutation and clonal expansion of B cells in the mDX400 group suggested that anti-PD-1 treatment may promote activation of humoral immunity. The activation of $\mathrm{B}$ cell responses may rely on interleukin-21 and $\mathrm{T}$ follicular helper cells induced by ICIs, as suggested by Hollern et al (15).

The BCR clone and the aforementioned consistently expanded TCR clone were co-expanded in $29 \%$ of the mDX400-group tumor samples, which may be related to tumor responses. Of note, the extreme expansion of the BCR clone may affect the expansion of the most frequent TCR clones, as the mouse with extreme expansion of the BCR clone was the only mouse without the high expansion of the top 100 most frequent TCR clones in the mDX400 group. The inhibitory 
effect of the increased expansion of the BCR clone on the expansion of high-frequency TCR clones needs to be further confirmed with larger sample sizes.

As for CDR3 lengths, the present study showed that applying anti-PD-1 had no effects on the CDR3 lengths of the TCR/BCR repertoires. Similarly, cytokine-based immunotherapy of murine CT26 colorectal tumors did not lead to significant changes of CDR3 length distribution (38). In addition, it has been reported that there is no correlation between the CDR3 length distributions and genetic variability of the CDR3 region (39), suggesting that the repertoire divergence between the treatment and control group in this study is not represented in terms of CDR3 length.

In summary, the effect of anti-PD-1 treatment on immune repertoires of the MC38 tumor model were elucidated. Anti-PD-1 treatment changed the usage frequency of some TRB VJ genes, decreased the diversity of intratumoral TCR repertoires, increased the proportion of intratumoral TCR clones that were shared between tumor and peripheral immune organs, and induced the expansion of a TCR and a BCR clone in $>50 \%$ of tumors. In addition, the consistently expanded TCR and BCR clone were co-expanded in $29 \%$ of anti-PD-1-group tumor samples. Anti-PD-1 treatment also upregulated the mutation rates of $\mathrm{IgH}$ sequences in spleen samples. These results showed that anti-PD-1 therapy promoted the activation of both cellular and humoral immunity in the MC38 model. The enhanced treatment efficacy in the MC38 model may be attributed to the enrichment of the putative tumor-reactive T and B cells, as well as increased BCR affinity in the spleen. As MC38 is a valid model for hypermutated and/or MSI CRC, it can be deduced that the molecular changes detected in the MC38 model may mirror those occurring in patients with hypermutated and/or MSI CRC. The decreased intratumoral TCR diversity, and the expansion of TCR and BCR clones may be used as biomarkers for prognostic rates in the clinical setting.

The present study also provided insights into novel treatment strategies. The significantly expanded intratumoral TCR clones induced by anti-PD-1 treatment may be reactive to tumor antigens, which opens the possibility of employing tumor-reactive $\mathrm{T}$ cell clones for adoptive $\mathrm{T}$ cell transfer as a passive immunization option. It has been demonstrated that the combination of adoptive $\mathrm{T}$ cell and anti-PD-1 immunotherapy potently enhances antitumor efficacy in a mouse model (40). There is also a possibility to develop cancer vaccines consisting of immunogenic peptides derived from antigens of poorly immunogenic tumors as an active immunization option. Vaccination with cancer vaccines may stimulate immune responses that will offset the inherently weak antigenicity of lowly immunogenic tumors. An early clinical trial testing the effect of a combination of anti-PD-1 antibodies with p53-expressing modified vaccinia Ankara virus vaccine in patients with advanced solid cancers showed encouraging results; it worked by increasing the frequency and persistence of $\mathrm{p} 53$-reactive $\mathrm{CD} 8^{+} \mathrm{T}$ cells (41). On the other hand, strategies modulating $\mathrm{B}$ cell responses may also have potential to improve treatment responses. Further investigation of the prognostic and therapeutic values of the molecular changes detected in the present study is warranted.

\section{Acknowledgements}

The authors would like to thank Principal Scientist Cai Li of Merck \& Co., Inc. (Kenilworth, NJ, USA) for helpful discussion and advice on the writing of the manuscript.

\section{Funding}

The present study was supported by Merck \& Co., Inc.

\section{Availability of data and materials}

The datasets used and/or analyzed during the present study are available from the corresponding author on reasonable request.

\section{Authors' contributions}

BL and NS conceived and designed the study, coordinated trials and provided data. IW coordinated the project and contributed key scientific insights in the analysis and interpretation of data. $\mathrm{RC}$ and GZ were involved in the analysis and interpretation of data. LZ analyzed the data and drafted the manuscript. All authors critically reviewed or revised the manuscript. All authors read and approved the final manuscript.

\section{Ethics approval and consent to participate}

The care and use of mice were reviewed and approved by Merck's Institutional Animal Care and Use Committee prior to conducting the study (approval no. 200321). During the study, the care and use of animals was conducted in accordance with the guidelines of the Association for Assessment and Accreditation of Laboratory Animal Care.

\section{Patient consent for publication}

Not applicable.

\section{Competing interests}

LZ, IW, NS, RC, GZ and BL are employees or former employees of Merck \& Co., Inc., Kenilworth, NJ, USA.

\section{References}

1. Hamid O, Robert C, Daud A, Hodi FS, Hwu WJ, Kefford R, Wolchok JD, Hersey P, Joseph R, Weber JS, et al: Five-year survival outcomes for patients with advanced melanoma treated with pembrolizumab in KEYNOTE-001. Ann Oncol 30: 582-588, 2019.

2. Gandhi L, Rodríguez-Abreu D, Gadgeel S, Esteban E, Felip E, De Angelis F, Domine M, Clingan P, Hochmair MJ, Powell SF, et al; KEYNOTE-189 Investigators: Pembrolizumab plus Chemotherapy in Metastatic Non-Small-Cell Lung Cancer. N Engl J Med 378: 2078-2092, 2018.

3. Bauml J, Seiwert TY, Pfister DG, Worden F, Liu SV, Gilbert J, Saba NF, Weiss J, Wirth L, Sukari A, et al: Pembrolizumab for platinum- and cetuximab-refractory head and neck cancer: Results from a single-arm, phase II study. J Clin Oncol 35: 1542-1549, 2017.

4. Sarfaty M, Hall PS, Chan KKW, Virik K, Leshno M, Gordon N, Moore A, Neiman V, Rosenbaum E and Goldstein DA: Cost-effectiveness of Pembrolizumab in Second-line Advanced Bladder Cancer. Eur Urol 74: 57-62, 2018. 
5. Carretero-González A, Lora D, Ghanem I, Zugazagoitia J, Castellano D, Sepúlveda JM, López-Martin JA, Paz-Ares L and de Velasco G: Analysis of response rate with ANTI PD1/PD-L1 monoclonal antibodies in advanced solid tumors: A meta-analysis of randomized clinical trials. Oncotarget 9: 8706-8715, 2018

6. Le DT, Durham JN, Smith KN, Wang H, Bartlett BR, Aulakh LK, Lu S, Kemberling H, Wilt C, Luber BS, et al: Mismatch repair deficiency predicts response of solid tumors to PD-1 blockade. Science 357: 409-413, 2017.

7. Yarchoan M, Hopkins A and Jaffee EM: Tumor Mutational burden and response rate to PD-1 inhibition. N Engl J Med 377: 2500-2501, 2017

8. Garon EB, Rizvi NA, Hui R, Leighl N, Balmanoukian AS, Eder JP, Patnaik A, Aggarwal C, Gubens M, Horn L, et al; KEYNOTE-001 Investigators: Pembrolizumab for the treatment of non-small-cell lung cancer. N Engl J Med 372: 2018-2028, 2015.

9. Ayers M, Lunceford J, Nebozhyn M, Murphy E, Loboda A, Kaufman DR, Albright A, Cheng JD, Kang SP, Shankaran V, et al: IFN- $\gamma$-related mRNA profile predicts clinical response to PD-1 blockade. J Clin Invest 127: 2930-2940, 2017.

10. Tumeh PC, Harview CL, Yearley JH, Shintaku IP, Taylor EJ, Robert L, Chmielowski B, Spasic M, Henry G, Ciobanu V, et al: PD-1 blockade induces responses by inhibiting adaptive immune resistance. Nature 515: 568-571, 2014

11. Roh W, Chen P-L, Reuben A, Spencer CN, Prieto PA, Miller JP, Gopalakrishnan V, Wang F, Cooper ZA, Reddy SM, et al: Integrated molecular analysis of tumor biopsies on sequential CTLA-4 and PD-1 blockade reveals markers of response and resistance. Sci Transl Med 9: eaah3560, 2017.

12. Minervina A, Pogorelyy $M$ and Mamedov I: T-cell receptor and B-cell receptor repertoire profiling in adaptive immunity. Transpl Int 32: 1111-1123, 2019.

13. Agata Y,Kawasaki A, Nishimura H,Ishida Y, Tsubata T, Yagita H and Honjo T: Expression of the PD-1 antigen on the surface of stimulated mouse T and B lymphocytes. Int Immunol 8: 765-772, 1996.

14. Thibult ML, Mamessier E, Gertner-Dardenne J, Pastor S, Just-Landi S, Xerri L, Chetaille B and Olive D: PD-1 is a novel regulator of human B-cell activation. Int Immunol 25: 129-137, 2013.

15. Hollern DP, Xu N, Thennavan A, Glodowski C, Garcia-Recio S, Mott KR, He X, Garay JP, Carey-Ewend K, Marron D, et al: B cells and $\mathrm{T}$ follicular helper cells mediate response to checkpoint inhibitors in high mutation burden mouse models of breast cancer. Cell 179: 1191-1206.e21, 2019.

16. Selitsky SR, Mose LE, Smith CC, Chai S, Hoadley KA, Dittmer DP, Moschos SJ, Parker JS and Vincent BG: Prognostic value of B cells in cutaneous melanoma. Genome Med 11: 36, 2019.

17. Helmink BA, Reddy SM, Gao J, Zhang S, Basar R, Thakur R, Yizhak K, Sade-Feldman M, Blando J, Han G, et al: B cells and tertiary lymphoid structures promote immunotherapy response. Nature 577: 549-555, 2020.

18. Olson B, Li Y, Lin Y, Liu ET and Patnaik A: Mouse Models for cancer immunotherapy research. Cancer Discov 8: 1358-1365, 2018

19. Hossain DMS, Javaid S, Cai M, Zhang C, Sawant A, Hinton M, Sathe M, Grein J, Blumenschein W, Pinheiro EM, et al: Dinaciclib induces immunogenic cell death and enhances anti-PD1-mediated tumor suppression. J Clin Invest 128: 644-654, 2018.

20. Ngiow SF, Young A, Jacquelot N, Yamazaki T, Enot D, Zitvogel L and Smyth MJ: A threshold level of intratumor CD8+ T-cell PD1 expression dictates therapeutic response to anti-PD1. Cancer Res 75: 3800-3811, 2015

21. Homet Moreno B, Zaretsky JM, Garcia-Diaz A, Tsoi J, Parisi G, Robert L, Meeth K, Ndoye A, Bosenberg M, Weeraratna AT, et al: Response to Programmed cell death-1 blockade in a murine melanoma syngeneic model requires costimulation, CD4, and CD8 T Cells. Cancer Immunol Res 4: 845-857, 2016.

22. Grasselly C, Denis M, Bourguignon A, Talhi N, Mathe D, Tourette A, Serre L, Jordheim LP, Matera EL and Dumontet C: The Antitumor activity of combinations of cytotoxic chemotherapy and immune checkpoint inhibitors is model-dependent. Front Immunol 9: 2100, 2018.

23. Efremova M, Rieder D, Klepsch V, Charoentong $P$, Finotello F, Hackl H, Hermann-Kleiter N, Löwer M, Baier G, Krogsdam A, et al: Targeting immune checkpoints potentiates immunoediting and changes the dynamics of tumor evolution. Nat Commun 9: 32, 2018

24. National Research Council (US) Committee for the Update of the Guide for the Care and Use of Laboratory Animals: Guide for the Care and Use of Laboratory Animals. 8th edition. National Academies Press, Washington, DC, 2011.
25. Masella AP, Bartram AK, Truszkowski JM, Brown DG and Neufeld JD: PANDAseq: Paired-end assembler for illumina sequences. BMC Bioinformatics 13: 31, 2012.

26. Giudicelli V, Chaume D and Lefranc MP: IMGT/GENE-DB: A comprehensive database for human and mouse immunoglobulin and T cell receptor genes. Nucleic Acids Res 33: D256-D261, 2005.

27. Ye J, Ma N, Madden TL and Ostell JM: IgBLAST: An immunoglobulin variable domain sequence analysis tool. Nucleic Acids Res 41 (W1): W34-40, 2013.

28. Gupta NT, Vander Heiden JA, Uduman M, Gadala-Maria D, Yaari G and Kleinstein SH: Change-O: A toolkit for analyzing large-scale B cell immunoglobulin repertoire sequencing data. Bioinformatics 31: 3356-3358, 2015.

29. Hill MO: Diversity and Evenness: A unifying notation and its consequences. Ecology 54: 427-432, 1973.

30. Shugay M, Bagaev DV, Zvyagin IV, Vroomans RM, Crawford JC, Dolton G, Komech EA, Sycheva AL, Koneva AE, Egorov ES, et al: VDJdb: A curated database of T-cell receptor sequences with known antigen specificity. Nucleic Acids Res 46 (D1): D419-D427, 2018.

31. Tickotsky N, Sagiv T, Prilusky J, Shifrut E and Friedman N: McPAS-TCR: A manually curated catalogue of pathology-associated T cell receptor sequences. Bioinformatics 33: 2924-2929, 2017.

32. Gros A, Robbins PF, Yao X, Li YF, Turcotte S, Tran E, Wunderlich JR, Mixon A, Farid S, Dudley ME, et al: PD-1 identifies the patient-specific $\mathrm{CD}^{+}$tumor-reactive repertoire infiltrating human tumors. J Clin Invest 124: 2246-2259, 2014.

33. Pasetto A, Gros A, Robbins PF, Deniger DC, Prickett TD, Matus-Nicodemos R, Douek DC, Howie B, Robins H, Parkhurst MR, et al: Tumor- and Neoantigen-reactive T-cell receptors can be identified based on their frequency in fresh tumor. Cancer Immunol Res 4: 734-743, 2016.

34. Hosoi A, Takeda K, Nagaoka K, Iino T, Matsushita H, Ueha S, Aoki S, Matsushima K, Kubo M, Morikawa T, et al: Increased diversity with reduced "diversity evenness" of tumor infiltrating T-cells for the successful cancer immunotherapy. Sci Rep 8: 1058,2018

35. Lehmann-Horn K, Wang SZ, Sagan SA, Zamvil SS and von Büdingen HC: B cell repertoire expansion occurs in meningeal ectopic lymphoid tissue. JCI Insight 1: e87234, 2016.

36. Katoh H, Komura D, Konishi H, Suzuki R, Yamamoto A, Kakiuchi M, Sato R, Ushiku T, Yamamoto S, Tatsuno K, et al: Immunogenetic profiling for gastric cancers identifies sulfated glycosaminoglycans as major and functional $\mathrm{b}$ cell antigens in human malignancies. Cell Rep 20: 1073-1087, 2017.

37. DeFalco J, Harbell M, Manning-Bog A, Baia G, Scholz A, Millare B, Sumi M, Zhang D, Chu F, Dowd C, et al: Non-progressing cancer patients have persistent B cell responses expressing shared antibody paratopes that target public tumor antigens. Clin Immunol 187: 37-45, 2018.

38. Probst P, Stringhini M and Neri D: Immunotherapy of CT26 murine tumors is characterized by an oligoclonal response against the AH1 tumor rejection antigen. bioRxiv: doi. org $/ 10.1101 / 789784$

39. Rock EP, Sibbald PR, Davis MM and Chien YH: CDR3 length in antigen-specific immune receptors. J Exp Med 179: 323-328, 1994.

40. John LB, Devaud C, Duong CP, Yong CS, Beavis PA, Haynes NM, Chow MT, Smyth MJ, Kershaw MH and Darcy PK: Anti-PD-1 antibody therapy potently enhances the eradication of established tumors by gene-modified T cells. Clin Cancer Res 19: 5636-5646, 2013.

41. Chung V, Kos FJ, Hardwick N, Yuan Y, Chao J, Li D, Waisman J, Li M, Zurcher K, Frankel P, et al: Evaluation of safety and efficacy of p53MVA vaccine combined with pembrolizumab in patients with advanced solid cancers. Clin Transl Oncol 21: 363-372, 2019.

This work is licensed under a Creative Commons Attribution-NonCommercial-NoDerivatives 4.0 International (CC BY-NC-ND 4.0) License. 